\title{
Calibração de objetivos na otimização da produção agrícola com recursos limitados
}

\author{
Pedro Marcio Ferreira ${ }^{1}$ (D), Sérgio Drumond Ventura ${ }^{1}$ (D) \& Angel Ramon \\ Sanchez Delgado ${ }^{1,2}$
}

(1) Universidade Federal Rural do Rio de Janeiro, Instituto de Ciências Exatas, Departamento de Matemática, Rodovia BR 465, Km 07, Zona Rural, Seropédica 23897-000, Rio de Janeiro, Brasil. E-mail: pedroborgesengen@gmail.com, ventura@ufrrj.br

(2) Universidade Federal Rural do Rio de Janeiro, Instituto de Ciências Exatas, Departamento de Matemática, Programa de Mestrado em Modelagem Matemática e Computacional, Rodovia BR 465, Km 07, Zona Rural, Seropédica 23897-000, Rio de Janeiro, Brasil. E-mail: asanchez@ufrrj.br

Ferreira P.M., Ventura S.D. \& Delgado A.R.S. (2020) Calibração de objetivos na otimização da produção agrícola com recursos limitados. Pesquisa e Ensino em Ciências Exatas e da Natureza, 4: e1460. http://dx.doi.org/10.29215/pecen.v4i0.1460

Editor acadêmico: Eudes Leite de Lima. Recebido: 15 janeiro 2020. Aceito: 16 julho 2020. Publicado: 24 julho 2020.

Resumo: A maximização da receita líquida com recursos limitados (água e terra) se torna uma boa opção na tomada de decisão para se chegar a um esquema ótimo de produção agrícola. Suponha conhecidas as soluções ótimas de programas lineares associados à maximização da receita líquida com recursos limitados de um conjunto de lotes de cultivo localizados em um mesmo município. Nesse trabalho, procura-se construir através da dualidade, um programa linear (PL) que represente os valores objetivos ótimos de cada lote; isto é, onde o erro relativo entre o valor objetivo ótimo do PL representativo e o valor objetivo ótimo de cada lote seja o menor possível (calibração). O procedimento computacional de calibração apresentado, foi testado satisfatoriamente para nove lotes localizados no perímetro irrigado de Gorutuba, município de Nova Porteirinha, norte do estado de Minas Gerais, mostrando assim, ser um esquema de calibração confiável.

Palavras chave: Agronegócio, dualidade, problema inverso.

Calibration of objetives in the optimization of agricultural production with limited resources

Abstract: The maximization of net revenue with limited resources (water and land) becomes a good option in decision making to arrive at an optimal agricultural production scheme. Suppose known the optimal solutions of linear programs associated with the maximization of the net revenue with limited resources of a set of cultivation plots located in the same municipality. This work seeks to build through duality, a linear program (PL) that represents the optimal objective values of each lot; that is, where the relative error between the optimal objective value of the representative PL and the optimal objective value of each lot is as small as possible (calibration). The computational calibration procedure presented, was tested satisfactorily for nine lots located in the irrigated perimeter of Gorutuba, municipality of Nova Porteirinha, northern Minas Gerais state, thus showing to be a reliable calibration scheme.

Key words: Agribusiness, duality, inverse problem.

\section{Introdução}

Estimar receitas líquidas agrícolas ótimas com insumos (ou recursos) limitados é um problema importante na tomada de decisões no agronegócio. A otimização da rentabilidade constitui um dos principais objetivos da empresa agrícola brasileira e está associada ao uso racional dos recursos disponíveis no processo de produção de forma a se obter os mais altos níveis de rendimento econômico. A água e a terra são fatores preponderantes para o êxito da 
agricultura e seu manejo racional é imperativo na otimização da produção agrícola (Delgado et al. 2010). Em um planejamento ótimo da produção agrícola com recursos (água e terra) limitados, procura-se selecionar as culturas e os meses de plantio que proporcionem a maximização da receita líquida e a melhor utilização dos recursos disponíveis (Frizzone et al. 2005).

Nas decisões, temos que escolher entre as diferentes políticas de produção, a mais eficiente em relação às metas e condições de viabilidade. As decisões baseadas no julgamento e na intuição podem ser satisfatórias quando o número de fatores do problema é limitado e suas relações são claras, mas em situações em que esse número cresce faz-se necessário a utilização de modelos matemáticos, os quais permitem representar alternativas ou simular condições reais.

A Programação Linear (PL) na Programação Matemática (Bazaraa et al. 1979) representa um dos modelos mais apropriados para resolver o problema fundamental da alocação ótima de recursos, visando encontrar a melhor distribuição e médias de produção disponíveis (Frizzone et al. 1997). O planejamento irracional das condições de área irrigada e o uso dos recursos justifica a utilização de técnicas de PL na procura da lucratividade das áreas irrigadas. Em geral, procurase construir um modelo de PL que possa selecionar as culturas a ser desenvolvidas e quanto de cada cultura deve ser plantada.

Nesse trabalho desenvolvemos e implementamos computacionalmente um procedimento baseado na teoria da dualidade de PL, capaz de gerar uma calibração confiável entre os valores objetivos ótimos de nove lotes localizados no perímetro irrigado de Gorutuba, município de Nova Porteirinha, norte do estado de Minas Gerais, e o valor objetivo ótimo de um PL representativo dos respectivos lotes considerados.

\section{Material e Métodos}

No planejamento agrícola, com limitações de água e terra, procura-se selecionar as culturas e os meses de plantio em um perímetro irrigado composto por um conjunto de lotes, que proporcionem a maximização da receita líquida e a melhor utilização dos recursos disponíveis. O problema é determinar um padrão de cultivo ótimo das culturas para cada lote, de tal maneira que a receita líquida seja máxima quando é feita uma racionalização dos recursos. Foram selecionados nove lotes com características de solo e sistemas de irrigação distintos, quatro culturas anuais: abóbora, feijão, milho, quiabo; e quatro culturas perenes: banana prata, banana nanica, limão e manga. Esquematicamente, o modelo de programação linear para representar o problema anterior, pode ser equacionado como segue:

$$
\begin{array}{lll}
\text { Maximizar } & \sum_{i=1}^{m} \sum_{j=1}^{12}\left(p_{i j} y_{i}-c_{i}\right) x_{i j}=\sum_{i=1}^{m} r_{i j} x_{i j} \\
\text { Sujeito a: } & \sum_{i=1}^{m} v_{i j} x_{i j} \leq V T_{j} & j=1, \ldots, 12 \\
& \sum_{i=1}^{m} x_{i j} \leq A T_{j} & j=1, \ldots, 12 \\
x_{i j} \geq 0 & i=1, \ldots, m, j=1, \ldots, 12
\end{array}
$$

Em que:

- $i$ - Representa a cultura agrícola, $i=1, \ldots, m$;

$\cdot j$ - Representa o mês do ano, $j=1, \ldots, 12$;

- $p_{i j}$ - Representa o preço da cultura cultura $i$ no mês $j\left(\mathrm{R} \$ \cdot \mathrm{kg}^{-1}\right)$;

- $y_{i}$ - Produtividade média da cultura $i\left(\mathrm{~kg} \cdot \mathrm{ha}^{-1}\right)$; 
- $c_{i}$ - A média dos custos médios da cultura $i\left(\mathrm{R} \$ \cdot \mathrm{ha}^{-1}\right)$;

- $x_{i j}$ - Área cultivada com a cultura $i$ no mês $j$ (ha);

- $v_{i j}$ - Volume de água utilizado com a cultura $i$ no mês $j\left(\mathrm{~m}^{3} \cdot \mathrm{ha}^{-1}\right)$;

- $V T_{j}$ - Volume total de água disponível no mês $j\left(\mathrm{~m}^{3}\right)$;

- $A T_{j}$ - Área total irrigável no mês $j$ (ha).

No que segue, considera-se dado um perímetro irrigado com $L$ lotes $(l=1,2, \ldots . L)$ de plantio, e a série de problemas de PL $\left(P P L^{l}\right)$, associados à produção de cada lote do perímetro:

$$
\begin{array}{lll}
\text { Maximizar } & \sum_{i=1}^{m} \sum_{j=1}^{12} r_{i j}^{l} x_{i j}^{l} & \\
\text { Sujeito a: } & \sum_{i=1}^{m} v_{i j}^{l} x_{i j}^{l} \leq V T_{j}^{l} & j=1,2, \ldots .12 \\
& \sum_{i=1}^{m} x_{i j}^{l} \leq A T_{j}^{l} & j=1,2, \ldots .12 \\
& x_{i j}^{l} \geq 0 &
\end{array}
$$

Onde $r_{i j}^{l}=p_{i j} y_{i}^{l}-c_{i}^{l}$, representa a receita líquida marginal da cultura $i$ no mês $j$, lote $l\left(R \$ h a^{-1}\right), y_{i}^{l}$ a produção média da cultura $i$ no lote $l, c_{i}^{l}$ o custo médio de produção da cultura $i$ no lote $l$. Note que o preço de cada cultura em cada mês, independe do lote. Mais ainda, $x_{i j}^{l}(h a)$ representa a área a ser plantada no lote $l$ da cultura $i$ no mês $j$. Aqui $v_{i j}^{l}$ representa o volume de água que precisa a cultura $i$ no mês $j$ para seu desenvolvimento no lote $l, V T_{j}^{l}$ o volume total de água disponível no lote $l$, durante o mês $j$, e $A T_{j}^{l}$ a área total irrigável no lote $l$, durante o mês $j$.

Suponha conhecidas $L$ soluções ótimas $\bar{x}^{l}=\left(\bar{x}_{i j}^{l}\right)$ da série $\left(P P L^{l}\right)$; isto é, $\bar{x}^{l}$ representa a tomada de decisão ótima para cada lote $l=1,2, \ldots L$. A meta do problema inverso de otimização aqui tratado é determinar um vetor $r=\left(r_{i j}\right)$, tal que as condições de otimalidade do programa não-linear:

$$
\begin{array}{lll}
\left(P^{l}\right) \quad \text { Maximizar } & \sum_{i=1}^{m} \sum_{j=1}^{12} r_{i j} x_{i j}^{l} & \\
\text { Sujeito a: } & \sum_{i=1}^{m} v_{i j}^{l} x_{i j}^{l} \leq V T_{j}^{l} & j=1,2, \ldots .12 \\
& \sum_{i=1}^{m} x_{i j}^{l} \leq A T_{j}^{l} & j=1,2, \ldots .12 \\
& x_{i j}^{l} \geq 0, r \in \mathbb{R}^{12 m} &
\end{array}
$$

sejam satisfeitas o mais aproximadamente possível pelas soluções ótimas $\bar{x}^{l}$, para cada $l=$ $1,2, \ldots . L$. Note que desta maneira, o vetor $r=\left(r_{i j}\right)$, representa devidamente as receitas $r^{l}=\left(r_{i j}^{l}\right)$ $\operatorname{dos} L$ lotes e permite avaliar economicamente a produtividade do perímetro irrigado através de $\left(P^{l}\right)$.

O nome de inverso, deve-se a que, neste caso, procura-se determinar os coeficientes da função objetivo do modelo, ao invés da solução ótima do problema. Matematicamente, neste trabalho, procuraremos desenvolver um procedimento computacional que seja capaz de gerar um vetor $r \in \mathbb{R}^{12 m}$, tal que para todo $l=1,2, \ldots . L$ :

$$
\frac{\left|r^{T} \bar{x}^{l}-r^{T} x^{*}\right|}{\left|r^{T} \bar{x}^{l}\right|}<\mu_{l},
$$

$r^{T} \bar{x}^{l} \neq 0, \mu_{l} \in(0,0.1)$ e $x^{* l}$ uma solução ótima de $\left(P^{l}\right)$. Assim teríamos que $r^{T} x^{* l}$ representa devidamente os valores objetivos ótimos dos $L$ lotes. 
Note que para cada $l=1,2, \ldots . L,\left(P^{l}\right)$ é um programa não linear nas variáveis $x_{i j}^{l} \in \mathbb{R}_{+}^{12 m}$, $r \in \mathbb{R}^{12 m}$, mas se $r$ fosse conhecido, o problema dual associado ao então programa linear $\left(P^{l}\right)$, estaria dado por:

$\left(D^{l}\right) \quad$ Minimizar $f\left(u^{l}, \xi^{l}\right)=\sum_{j=1}^{12} V T_{j}^{l} u_{j}^{l}+\sum_{j=1}^{12} A T_{j}^{l} \xi_{j}^{l}$

Sujeito $a: v_{i j}^{l} u_{j}^{l}+\xi_{j}^{l}-z_{i j}^{l}=r_{i j} i=1,2, \ldots . . m ; j=1,2, \ldots .12$

$$
u_{j}^{l}, \xi_{j}^{l}, z_{i j}^{l} \geq 0
$$

Também o $\left(P^{l}\right)$ pode ser escrito como:

$$
\begin{gathered}
\text { Maximizar } \sum_{i=1}^{m} \sum_{j=1}^{12} r_{i j} x_{i j}^{l} \\
\text { Sujeito a: } \sum_{i=1}^{m} v_{i j}^{l} x_{i j}^{l}+w_{j}^{1 l}=V T_{j}^{l} \quad j=1,2, \ldots .12 \\
\sum_{i=1}^{m} x_{i j}^{l}+w_{j}^{2 l}=A T_{j}^{l} \quad j=1,2, \ldots .12 \\
x_{i j}^{l} \geq 0, w_{j}^{1 l} \geq 0, w_{j}^{2 l} \geq 0
\end{gathered}
$$

Da teoria de dualidade, se $x^{l}$ é primal viável e $\left(u^{l}, \xi^{l}\right)$ dual viável, então:

$$
f\left(u^{l}, \xi^{l}\right)-r^{T} x^{l}=\left(z^{l}\right)^{T} x^{l}+\left(w^{1 l}\right)^{T} u^{l}+\left(w^{2 l}\right)^{T} \xi^{l} \geq 0 ; \text { ou } r^{T} x^{l} \leq f\left(u^{l}, \xi^{l}\right)
$$

(teorema fraco de dualidade) e a igualdade é alcançada nas soluções ótimas primal-dual. Por isso, a construção do modelo para resolver o problema inverso de otimização, incorpora a restrição contrária: $r^{T} x^{l} \geq f\left(u^{l}, \xi^{l}\right)$, ao sistema de desigualdades viáveis primal-dual; isto é, para cada $l=1,2, \ldots . L$, procuram-se vetores $x^{l}$ e $\left(u^{l}, \xi^{l}\right)$ tais que:

$$
\begin{gathered}
f\left(u^{l}, \xi^{l}\right)-r^{T} x^{l} \leq 0 \\
\sum_{i=1}^{m} v_{i j}^{l} x_{i j}^{l} \leq V T_{j}^{l} \quad j=1,2, \ldots .12 \\
\sum_{i=1}^{m} x_{i j}^{l} \leq A T_{j}^{l} \quad j=1,2, \ldots .12 \\
r_{i j}-v_{i j}^{l} u_{j}^{l}-\xi_{j}^{l} \leq 0 \quad i=1,2, \ldots . m ; j=1,2, \ldots .12
\end{gathered}
$$

Voltando a nossa visão de $r$ e $u^{l}, \xi^{l}$ (multiplicadores de Lagrange) como variáveis, ao substituir $x_{i j}^{l}$ por $\bar{x}_{i j}^{l}$ em (1)-(3), temos que (2)-(3) são trivialmente cumpridas e por isso resta agora o conjunto de desigualdades dadas por (1) e (4), ou seja, para cada $l=1,2, \ldots . L$ :

$$
\begin{gathered}
f\left(u^{l}, \xi^{l}\right)-\left(\bar{x}^{l}\right)^{T} r \leq 0 \\
r_{i j}-v_{i j}^{l} u_{j}^{l}-\xi_{j}^{l} \leq 0 \quad i=1,2, \ldots . . m ; j=1,2, \ldots .12
\end{gathered}
$$

Este é um conjunto de $L(12 m+1)$ desigualdades lineares e $12(m+2 L)$ variáveis. Para efeitos numéricos, suponha que $L$ é um inteiro positivo estritamente maior que $\frac{12 m}{12 m-23}$, assim existirão mais desigualdades do que variáveis. Na prática, em lugar de encontrar uma solução do sistemas de desigualdades (4)-(5), determina-se uma solução ótima do problema auxiliar de programação linear dado por:

$$
\text { Minimizar } \sum_{l=1}^{L} \alpha_{l}
$$

Sujeito a: $\sum_{j=1}^{12} V T_{j}^{l} u_{j}^{l}+\sum_{j=1}^{12} A T_{j}^{l} \xi_{j}^{l}-\sum_{i=1}^{m} \sum_{j=1}^{12} \bar{x}_{i j}^{l} r_{i j}+\alpha_{l} \leq 0, \quad l=1,2, \ldots . L$

$$
r_{i j}-v_{i j}^{l} u_{j}^{l}-\xi_{j}^{l} \leq 0 \quad i=1,2, \ldots . . m ; j=1,2, \ldots .12, \quad l=1,2, \ldots . L
$$




$$
\begin{array}{ll}
0<\alpha_{l}<1 & l=1,2, \ldots . L \\
u^{l}, \xi^{l} \geq 0, r \in \mathbb{R}^{12 m} & l=1,2, \ldots . L
\end{array}
$$

Note que uma solução ótima de $(P A)$, gera vetores $r, u^{l}, \xi^{l}$ tais que (4)-(5)- são satisfeitas. Agora, depois que a essência do problema e a respectiva teoria para seu tratamento foram apresentadas, se faz necessário detalhar um procedimento computacional para a utilização do modelo.

\section{Procedimento}

Dados: $m \geq 2, L$ inteiro positivo estritamente maior que $\frac{12 m}{12 m-23}, p_{i j}, y_{i}^{l}, c_{i}^{l}, v_{i j}^{l}, V T_{j}^{l}, A T_{j}^{l} \operatorname{com} i=$ $1,2, \ldots . m, j=1,2, \ldots .12, \quad l=1,2, \ldots . L$ (indicadores).

\section{Começo}

\section{PASSO 1}

Para cada $l=1,2, \ldots . L$

Para cada $i=1,2, \ldots . m$

Para cada $j=1,2, \ldots 12$

Calcular $r^{l}=\left(r_{i j}^{l}\right)$ onde $r_{i j}^{l}=p_{i j} y_{i}^{l}-c_{i}^{l}$

Para cada $\quad l=1,2, \ldots . L$

Fazer

$$
\bar{x}^{l}=\operatorname{Argmax}\left\{\begin{array}{c}
\left(r^{l}\right)^{T} x^{l}: \sum_{i=1}^{m} v_{i j}^{l} x_{i j}^{l} \leq V T_{j}^{l}, \sum_{i=1}^{m} x_{i j}^{l} \leq A T_{j}^{l}, x_{i j}^{l} \geq 0, \\
j=1,2, \ldots .12
\end{array}\right\}
$$

\section{PASSO 2}

\section{Resolver $(P A)$}

Saída: $\left(r, u^{l}, \xi^{l}, \alpha_{l}\right)$

\section{PASSO 3}

Para cada $\quad l=1,2, \ldots . L$

\section{Fazer}

$$
x^{* l}=\operatorname{Argmax}\left\{\begin{array}{c}
r^{T} x^{l}: \sum_{i=1}^{m} v_{i j}^{l} x_{i j}^{l} \leq V T_{j}^{l}, \sum_{i=1}^{m} x_{i j}^{l} \leq A T_{j}^{l}, x_{i j}^{l} \geq 0, \\
j=1,2, \ldots .12
\end{array}\right\}
$$

Para cada $\quad l=1,2, \ldots . L$

Enquanto $\gamma_{l}=r^{T} \bar{x}^{l} \neq 0$ 
Avaliar erro $=\frac{\left|r^{T} \bar{x}^{l}-r^{T} x^{*}\right|}{\left|r^{T} \bar{x}^{l}\right|}$

Fim

O procedimento anterior é uma maneira de se determinar um vetor objetivo que representa aproximadamente os objetivos dos $L$ lotes de plantios dados. No PASSO 1, gera-se a série de problemas associados aos $L$ lotes. No PASSO 2, se resolve o PL auxiliar (PA), obtendo-se o vetor objetivo $r$, os multiplicadores de Lagrange, $u^{l}, \xi^{l}$ e as variáveis $\alpha_{l}$, que medem o grau de aproximação da solução primal-dual gerada pelo procedimento (para cada $l=1,2, \ldots . L$ ). No PASSO 3, encontra-se uma solução ótima do problema de PL com o vetor objetivo $r$ (determinado no PASSO 2) e logo realizamos uma avaliação do erro relativo entre o valor $r^{T} x^{* l}$ e o valor ótimo do $\left(P^{l}\right)$, agora com $r$ representando os $r^{l}$. A implementação do procedimento foi realizada utilizando MATLAB.

O material ou dados utilizados no ensaio numérico a ser apresentados na próxima seção, correspondem ao perímetro irrigado de Gorutuba, localizado no município de Nova Porteirinha, norte do estado de Minas Gerais e descrito em Carvalho (1998) e Carvalho et al. (2000). Da mesma forma que o referido autor, nove lotes foram selecionados $(l=1,2, \ldots . L=9)$ por apresentarem características de solo e sistemas de irrigação distintos. A escolha das culturas baseou-se na maior variedade, dentre aqueles implantados e com a maior diversidade de características físico-hídricas dos solos. Foi simulado o plantio de quatro culturas anuais: abóbora, feijão, milho, quiabo; e de quatro culturas perenes: banana prata, banana nanica, limão e manga $(i=1,2, \ldots . m=8)$.

\section{Resultados e Discussão}

A Tabela 1 apresenta os valores dos parâmetros: preços, produtividade média e custos das culturas anuais e perenes utilizados. $\mathrm{Na}$ obtenção dos custos totais $\left(\mathrm{R} \$ \cdot \mathrm{ha}^{-1}\right)$ para cada cultura, além dos valores na tabela, foi considerado o valor da tarifa de água cobrado no perímetro segundo informações do distrito de irrigação de Gorutuba. É importante ressaltar que a vigência dos valores desses parâmetros não foi considerada relevante, posto que o maior interesse do trabalho foi testar computacionalmente a confiabilidade do procedimento de calibração apresentado na seção anterior.

Em função dessas ofertas, as datas de plantio para as culturas anuais foram adotadas, considerando a duração média do ciclo da cultura. Para as culturas perenes, foram considerados, ao invés do mês de plantio, os meses em que a cultura estivesse produzindo, sendo o produto vendido àquele preço apresentado na Tabela 1 . Dessa maneira, não se estava buscando qual a área a ser plantada e sim, qual a área que deveria estar produzindo em determinado mês com determinada cultura. Como não foi considerada a duração do ciclo da cultura, a produtividade média adotada para cada mês foi inversamente proporcional ao preço observado na região naquele mês, respeitando, dessa forma, a lei da oferta e da procura.

Nesta experiência numérica, estaremos supondo que o custo médio de produção de cada cultura agrícola, $c_{i}$, é o mesmo em qualquer lote, $l=1,2, \ldots . L$; isto é, $c_{i}^{l}=c_{i}$. Observando a Tabela 1, verificam-se os meses em que a oferta varia ao longo do ano e em função destas ofertas e duração média do ciclo de cada cultura, foram consideradas as datas de plantio das culturas anuais. Por exemplo, o feijão apresenta preços competitivos em nove meses do ano, sendo considerado como mês de plantio aquele que antecedia em três meses o mês com aquele preço. Por isso, foram considerados como meses de plantio: janeiro, fevereiro, março, abril, maio, julho, agosto, outubro e novembro, totalizando nove épocas. O mesmo foi feito para as outras culturas anuais, obtendo-se um mês de plantio para a abóbora, sete para o milho e dois para o quiabo.

A Tabela 2 apresenta as produtividades médias de cada cultura em cada lote $\left(y_{i}^{l}\right)$ (variantes da produtividade média de cada cultura $\left(y_{i}\right)$ dada na Tabela 1).

Já a Tabela 3, mostra os volumes mensais de água necessários para o desenvolvimento de cada cultura considerada. Também nesta experiência numérica, estaremos supondo que o 


\section{Otimização da produção agrícola}

volume mensal necessário para o desenvolvimento de qualquer das culturas é o mesmo em qualquer lote $l=1,2, \ldots . L=9$; isto é: $v_{i j}^{l}=v_{i j}$.

Tabela 1. Preços das culturas agrícolas $\left(p_{i j}\right)$, produtividade média $\left(y_{i}\right)$ e custos médios de produção $\left(c_{i}\right)$ para as culturas consideradas, no perímetro irrigado de Gorutuba (MG).

\begin{tabular}{|c|c|c|c|c|c|c|c|c|}
\hline & Abóbora & Feijão & Milho & Quiabo & Banana Prata & Banana Nanica & Limão & Manga \\
\hline Mês & \multicolumn{8}{|c|}{ Preço das culturas agrícolas $\left(\mathbf{R} \$ \cdot \mathbf{k g}^{-1}\right)$} \\
\hline Janeiro & & 0.662 & & & 0.401 & 0.084 & 0.307 & \\
\hline Fevereiro & & 0.500 & 0.180 & 0.400 & 0.484 & 0.085 & 0.188 & \\
\hline Março & & & 0.154 & & 0.375 & 0.158 & 0.180 & \\
\hline Abril & & 0.750 & 0.148 & & 0.404 & 0.126 & 0.180 & \\
\hline Maio & & 0.831 & 0.156 & & 0.431 & 0.091 & 0.364 & \\
\hline Junho & & 0.830 & & & 0.601 & 0.090 & & \\
\hline Julho & & 0.829 & 0.161 & & 0.592 & 0.092 & & \\
\hline Agosto & & 0.756 & 0.164 & 0.455 & 0.413 & 0.219 & 0.402 & 1.00 \\
\hline Setembro & & & & & 0.315 & 0.182 & 0.751 & \\
\hline Outubro & & 0.818 & & & 0.214 & 0.269 & 0.751 & \\
\hline Novembro & 0.20 & 0.752 & 0.156 & & 0.215 & 0.158 & 0.384 & 0.294 \\
\hline \multirow[t]{5}{*}{ Dezembro } & & & & & 0.198 & 0.092 & 0.429 & 0.219 \\
\hline & \multicolumn{8}{|c|}{ Produtividade $\left(\mathrm{kg} \cdot \mathrm{ha}^{-1}\right)$} \\
\hline & 18.000 & 1.800 & 5.500 & 15.000 & 26.000 & 44.000 & 22.000 & 18.000 \\
\hline & \multicolumn{8}{|c|}{ Custo de produção $\left(\mathbf{R} \$ \cdot \mathbf{h a}^{-1}\right)$} \\
\hline & 2.674 .20 & 965.00 & 651.50 & 3.611 .50 & 1951.5 & 2.676 .5 & 996.40 & 1.615 .90 \\
\hline
\end{tabular}

Tabela 2. Produtividade média $\left(\mathrm{kg} \cdot \mathrm{ha}^{-1}\right)$ de cada cultura em cada lote do perímetro irrigado de Gorutuba$\operatorname{MG}\left(y_{i}^{l}\right)$.

\begin{tabular}{lccccccccc}
\hline & \multicolumn{7}{c}{ Lotes } \\
\hline Culturas & $\mathbf{1}$ & $\mathbf{2}$ & $\mathbf{3}$ & $\mathbf{4}$ & $\mathbf{5}$ & $\mathbf{6}$ & $\mathbf{7}$ & $\mathbf{8}$ & $\mathbf{9}$ \\
\hline Abóbora & 18.000 & 9.000 & 6.000 & 4.500 & 3.600 & 3.000 & 2.571 .42 & 2.250 & 2.000 \\
Feijão & 1.800 & 900 & 600 & 450 & 360 & 300 & 257.14 & 225 & 200 \\
Milho & 5.500 & 2.250 & 1.833 .33 & 1.375 & 1.100 & 916.66 & 785.71 & 687.50 & 611.11 \\
Quiabo & 15.000 & 7.500 & 5.000 & 3.750 & 3.000 & 2.500 & 2.142 .85 & 1.875 & 1.666 .66 \\
Banana Prata & 26.000 & 13.000 & 8.666 .66 & 6.500 & 5.200 & 4.333 .33 & 3.714 .28 & 3.250 & 2.888 .88 \\
Banana Nanica & 44.000 & 22.000 & 14.666 .66 & 11.000 & 8.800 & 7.333 .33 & 6.285 .71 & 5.500 & 4.888 .88 \\
Limão & 22.000 & 11.000 & 7.333 .33 & 5.500 & 4.400 & 3.666 .66 & 3.142 .85 & 2.750 & 2.444 .44 \\
Manga & 18.000 & 9.000 & 6.000 & 4.500 & 3.600 & 3.000 & 2.571 .42 & 2.250 & 2.000 \\
\hline
\end{tabular}

Tabela 3. Volume de água mensal necessário para o desenvolvimento de cada cultura no perímetro irrigado de Gorutuba (MG). $\left(v_{i j}\right)$.

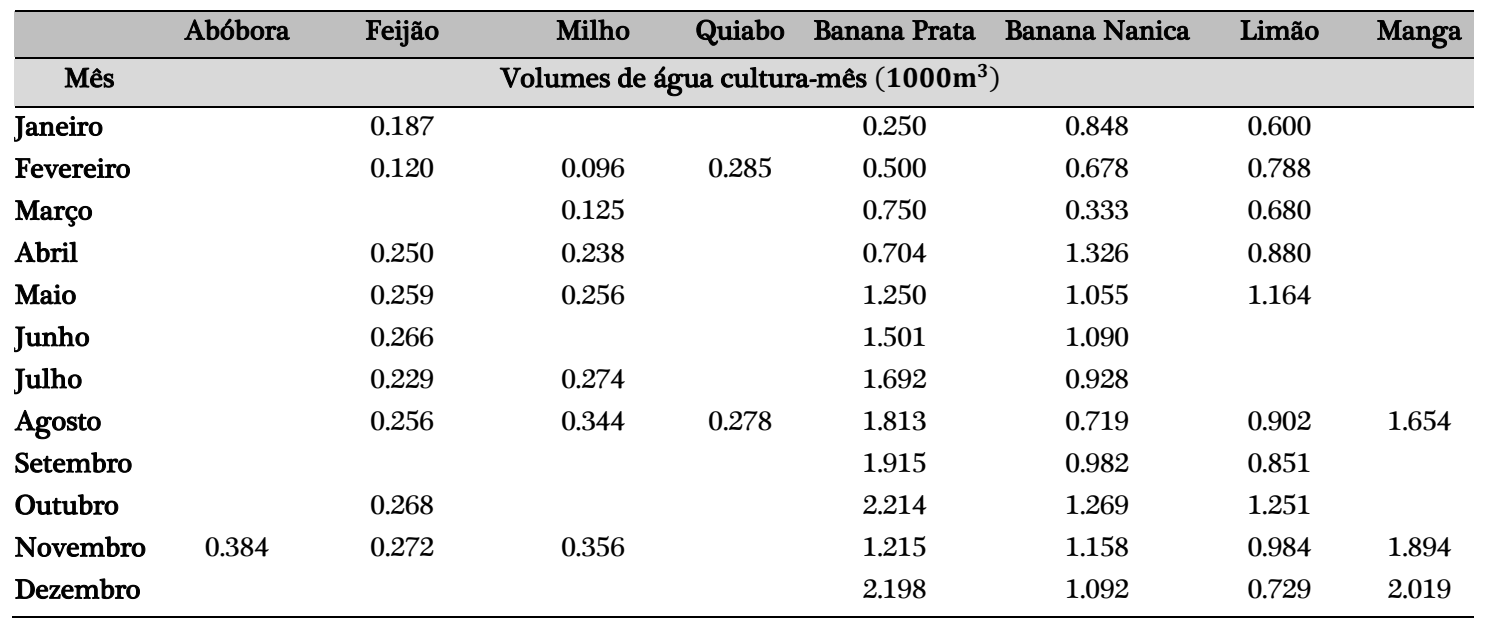

A Tabela 4 apresenta para cada lote $l$, os volumes de água disponíveis em cada mês do ano e as áreas irrigáveis em cada lote respectivamente $\mathrm{O}$ volume total de água disponível para cada lote $l$, no mês $j\left(V T_{j}^{l}\right)$, foi considerado igual àquele fornecido para os lotes em cada mês ao 


\section{Otimização da produção agrícola}

longo do ano, e a área total irrigável $\left(A T_{j}^{l}\right)$ foi ponderada como sendo a área irrigável de cada lote, ou seja, a área máxima possível de ser ocupada com o cultivo, no lote $l$ e mês $j$ (Carvalho et al. 2000).

Para todos os lotes foi considerada a área máxima possível de ser ocupada com o cultivo. Foram escolhidos entre cinco e nove lotes de plantio e a Tabela 5 mostra os resultados obtidos.

Tabela 4. Volumes mensais de água $\left(V T_{j}^{l}\right)$ em $1000 \mathrm{~m}^{3}$ e área irrigável $\left(A T_{j}\right)$ em ha para os lotes estudados.

\begin{tabular}{|c|c|c|c|c|c|c|c|c|c|}
\hline Lotes $(l)$ & 1 & 2 & 3 & 4 & 5 & 6 & 7 & 8 & 9 \\
\hline Mês & \multicolumn{9}{|c|}{ Volumes Mensais $\left(1000 \mathrm{~m}^{3}\right)$} \\
\hline Janeiro & 53.10 & 11.09 & 12.35 & 5.15 & 5.29 & 6.35 & 13.36 & 6.55 & 12.35 \\
\hline Fevereiro & 85.82 & 17.42 & 8.86 & 17.93 & 8.91 & 9.34 & 17.82 & 7.88 & 17.82 \\
\hline Março & 59.80 & 11.09 & 5.74 & 11.38 & 05.81 & 5.74 & 12.17 & 3.92 & 11.48 \\
\hline Abril & 60.26 & 14.26 & 7.06 & 7.16 & 7.06 & 7.06 & 14.11 & 6.88 & 14.44 \\
\hline Maio & 88.02 & 17.42 & 8.78 & 8.78 & 17.68 & 8.78 & 17.93 & 8.68 & 15.92 \\
\hline Junho & 75.29 & 15.84 & 15.84 & 7.92 & 8.10 & 7.92 & 9.70 & 8.10 & 13.08 \\
\hline Julho & 71.14 & 15.84 & 15.84 & 7.92 & 8.35 & 8.35 & 15.60 & 7.85 & 14.19 \\
\hline Agosto & 81.00 & 16.63 & 7.92 & 7.87 & 8.30 & 8.30 & 14.61 & 9.11 & 14.95 \\
\hline Setembro & 76.32 & 16.42 & 7.35 & 7.49 & 7.49 & 7.49 & 14.98 & 8.93 & 13.33 \\
\hline Outubro & 52.81 & 12.10 & 10.62 & 5.18 & 2.66 & 5.31 & 10.26 & 6.55 & 8.32 \\
\hline Novembro & 19.08 & 4.32 & 3.96 & 1.98 & 1.80 & 1.99 & 3.96 & 2.59 & 2.31 \\
\hline Dezembro & 18.00 & 6.05 & 4.54 & 2.27 & 1.95 & 2.95 & 5.29 & 2.23 & 2.89 \\
\hline \multicolumn{10}{|c|}{ Área (ha) } \\
\hline & 38.00 & 11.00 & 8.46 & 3.51 & 5.94 & 2.95 & 8.16 & 5.90 & 7.97 \\
\hline
\end{tabular}

Tabela 5. Resultados obtidos da implantação computacional do procedimento apresentado.

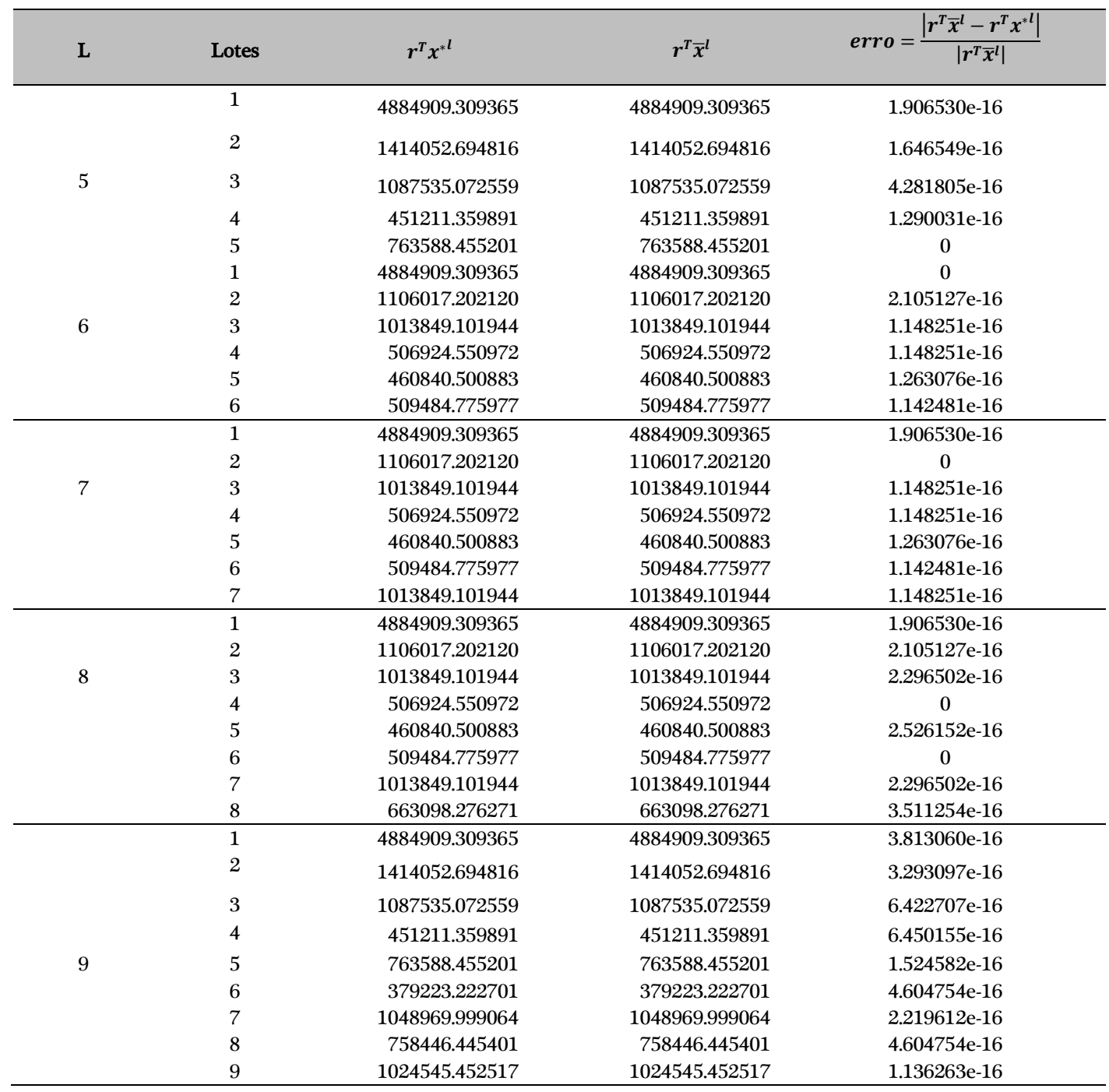




\section{Otimização da produção agrícola}

\section{Agradecimentos}

Os autores agradecem aos avaliadores pela revisão crítica do manuscrito.

\section{Conclusões}

O procedimento computacional implementado mostra um bom comportamento se observamos os resultados obtidos. Os erros relativos entre o valor $r^{T} x^{* l}$ e o valor ótimo do $\left(P^{l}\right)$, agora com $r$ representando os $r^{l}$, são satisfatórios, e portanto, o objetivo do trabalho apresentado é alcançado.

\section{Referências}

Bazaraa M.C., Sherali H.D. \& Shetty H.D. (1979) Nonlinear Programming: Theory and Algorithms. New York: John Wiley. 638 p.

Carvalho D.F. (1998) Otimização do uso da água no perímetro irrigado do Gorutuba. Tese (Programa de Pós-Graduação em Engenharia Agrícola), Universidade Federal de Viçosa, Viçosa, Minas Gerais.

Carvalho D.F., Soares A., Ribeiro C.A., Sediyama C.G. \& Pruski F. (2000) Otimização do uso da água no perímetro irrigado do Gorutuba, utilizando-se a técnica da programação linear. Revista Brasileira de Engenharia Agrícola e Ambiental, 4(2): 203-209. https://doi.org/10.1590/S1415-43662000000200012

Delgado A.R.S., Ventura S.D., Carvalho D.F. \& Santos R.D. (2010) Determinação de intervalos ótimos de irrigação utilizando barreira logarítmica. Revista Brasileira de Agricultura Irrigada, 4(2): 128-138. https://doi.org/10.7127/rbai.v4n200030

Frizzone J.A., Andrade A.S., de Souza J.L.M. \& Zocoler J.L. (2005) Planejamento de irrigação. Análise de decisão de investimentos. Brasília: EMBRAPA. 627 p.

Frizzone J.A., Coelho R.D., Dourado D.N. \& Soliane R. (1997) Linear programming model to optimize the water resource use in irrigation projects: an application to the Senator Nilo Coelho Project. Scientia Agricola, 54(especial): 136-148.

https://doi.org/10.1590/S0103-90161997000300016 